\title{
The Value of Prolonged Electroencepha- lographic and Video Monitoring in Diagnosis of Seizure Disorders
}

\author{
J.P. Willmer and D.G. Brunet
}

\begin{abstract}
Prolonged electroencephalographic and video monitoring of ictal events is useful in aiding the diagnosis of complicated seizure disorders. Forty eight patients admitted to a neurology service were assessed with this technique. Twenty five (54\%) had their diagnoses changed, six (14\%) had their diagnoses confirmed and the remaining patients had inconclusive studies. Therapy is modified by the results and in addition both patient and staff education is enhanced through the use of this equipment.
\end{abstract}

\begin{abstract}
RÉSUMÉ: Evaluation de l'électroencéphalographie continue et de la surveillance par vidéo dans le diagnostic des affections convulsives L'électroencéphalographie continue et la surveillance par vidéo des crises est un outil utile au diagnostic des affections convulsives complexes. Quarante-huit patients admis dans un service de neurologie ont été évalués avec cette technique. Chez vingt-cinq patients $(54 \%)$ on a changé le diagnostic à la suite de ces études et chez six on a confirmé le diagnostic. Pour le reste des patients, les études n étaient pas concluantes. La thérapie est modifiée par les résultats de ces études et l'utilisation de cet équipement est un moyen d'éducation efficace tant pour les patients que pour le personnel.
\end{abstract}

Can. J. Neurol. Sci. 1986: 13:327-330

The availability of equipment that provides reliable multichannel electroencephalographic (EEG) telemetry and simultaneous video monitoring has greatly improved the accuracy of diagnosis, and the treatment of patients with complicated seizure disorders. We wished to determine the effectiveness of this procedure in this patient population. A review of all patients seen at the Kingston General Hospital for prolonged EEG and video monitoring from the time this technique was instituted in July 1984 until July 1985 was undertaken. The indications for monitoring, diagnostic accuracy and therapeutic implications of the procedure were examined.

This technique has previously been reported in routine diagnostic work with some success. ${ }^{1,2}$ Intensive monitoring has been applied to the diagnosis of pseudoseizures, both in patients with co-existent epilepsy and those without..$^{3-9}$ In addition to differentiating pseudoseizures from epileptic seizures, intensive monitoring may aid in more accurate classification of seizure type and hence may improve treatment. This seems particularly true in distinguishing partial complex seizures from absence seizures. ${ }^{10}$ Further insights into the management of refractory epilepsy may be gained by combining intensive EEG and video monitoring with repeated anticonvulsant serum level determinations. ${ }^{11-14}$ Intensive monitoring may aid in determining the efficacy of therapy. Ictal and interictal behavioural disturbances have been studied with this technique. ${ }^{15.16}$ It may aid in the preoperative work up of epileptic patients by helping to determine localization of seizure discharges. ${ }^{17}$ These techniques have been applied effectively in the pediatric age group, ${ }^{5.18 .19}$ as well as in adults.

Seizures may be modified by environmental factors and circadian rhythms. ${ }^{20}$ Emotions, stress and hormone levels may all play a role in precipitating them. Seizure threshold varies during the normal sleep-wake cycle, and many patients have more seizures at night or immediately on arousal. Intensive EEG and video monitoring allows one to look at both environmental factors and the effects of the normal diurnal cycle.

\section{Methods}

\section{Patients}

A total of 48 patients were admitted to the neurology service at Kingston General Hospital for study. Of these patients, 28 $(58 \%)$ were female and $20(42 \%)$ male. Their ages ranged from 13 to 57 years with a mean age of 30 years. The pre-assessment diagnoses are outlined in Table 1 . The most common diagnosis was partial complex seizures (75\%) with more females (48\%) than males $(27 \%)$. 
Table 1: Premonitoring Diagnosis

\begin{tabular}{lccc}
\hline \hline Seizure Type & Male & Female & Total \\
\hline Generalised tonic clonic & $4(8)^{*}$ & $4(8)$ & 8 \\
Partial complex & $13(27)$ & $23(48)$ & 36 \\
Pseudoseizures & $1(2)$ & $1(2)$ & 2 \\
Not applicable & $2(5)$ & $0(0)$ & 2 \\
\hline Total & $20(42)$ & $28(58)$ & 48 \\
\hline
\end{tabular}

*Figures in brackets represent rounded percentages.

Most of the patients had had previous EEG recordings, ranging in number from none to 31 . Fourteen patients had had more than five. Many patients had had multiple hospital admissions for both assessment and treatment of seizures.

Thirty three percent of the patients had similar psychosocial backgrounds consisting of previous child abuse, poor marital and extramarital relationships and trouble maintaining employment. Many (74\%) had no underlying pathology as a presumed cause of their seizures. Fifteen percent had past histories of previous head injuries sufficient to cause loss of conciousness. Twenty six percent had pathological correlates of their seizure disorders, or a previously diagnosed neurological illness (Table 2), that was likely to result in a seizure disorder.

\section{Technique}

Patients were monitored with radiotelemetry EEG recording and simultaneous video recording using a Modac sixteen channel device produced by Telefactor Corporation. The EEG record was available continuously during the patient's admission provided that the patient stayed within the confines of the ward. The video record was only available while the patient was in the monitoring room.

An alarm button was provided at the bedside which logged the time when pushed. Patients were instructed in the use of this, as were the nursing and house staff of the neurology ward. The logged alarms and completed video tapes were collected twice a day. At these times the electrodes were also checked. In addition, the nursing staff and sometimes the patient were instructed in how to aim and adjust the camera.

All patients were recorded using a sixteen channel bipolar antero-posterior montage. The electrode placement was in accordance with the International 10-20 System. Some patients were also recorded using sixteen channel bipolar coronal montages, with or without extra temporal electrodes, $\mathrm{T} 1$ and $\mathrm{T} 2$. The electrodes were fastened to the scalp with collodion, and then filled with conductive gel. The wires were fastened together and connected to the transmitter, which was contained in a nylon harness worn over the patient's shoulders. This arrangement was usually effective for twenty four hour periods.

The EEG and video image were combined by a video reformatter, displayed in a split screen format on a monitor, and recorded on standard video cassettes. An automatic changer allowed 24 hours of unattended recording. Occasional jamming of the cassettes occurred and the nursing staff were taught how to detect and correct this problem.

Activation procedures were used in some of the patients. Anticonvulsant medication was either fully or partially withdrawn prior to or during the recording session. Sleepdeprivation, alcohol, hyperventilation and suggestion were all used in small numbers of these patients.
Table 2: Diagnoses in 12 Patients with History of Previous Neurological Illness

Subdural hematoma, craniotomy

Hemophilus meningitis, craniotomy.

Spinocerebellar degeneration, cerebellar biopsy.

Congenital hemiparesis.

Midline, intra-axial, low grade glioma.

Subdural hematoma, craniotomy, viral meningitis.

Toxemia of pregnancy, prolonged coma, seizures.

Subdural hematoma, craniotomy, Von Willebrand's disease.

Arachnoid cyst, craniotomy, residual porencephaly.

Low grade temporal lobe glioma.

Perinatal intraventricular hemmorhage.

Western Equine encephalitis.

Recording was usually for a full four day period. If it was felt that sufficient information had been gathered earlier, then the recording was stopped. In one patient, two four day periods were necessary.

All the records were reviewed weekly by one of the authors. Attempts were made to classify the ictus according to the International Classification of Epileptic Seizures as outlined by Dreifuss. ${ }^{21}$ Both clinical and EEG criteria were applied. In order to differentiate seizures of psychogenic origin, the criteria set forth by Desai, Porter and Penry ${ }^{7}$ were used. The major criteria included features such as bizzare and varied behaviour as opposed to sterotyped actions, EEG tracings that were normal or unchanged during the ictus, no postictal slowing of the EEG record and no relationship of frequency of symptoms to the patient's medication regime. The records were interpreted conservatively, as it is recognized that clinical seizures may occur in the absence of scalp EEG changes. ${ }^{22}$ At least one typical clinical event coupled with reliable EEG changes, or more than one typical clinical event with no EEG changes were required to make a diagnosis. If these criteria were not met, then the recording was classified as inconclusive. In addition, previous hardcopy EEG recordings were reviewed. One patient also had supplementary out-patient ambulatory four channel cassette recording (Oxford Medilog) on two occasions.

\section{RESULTS}

Two patients were excluded from the analyses as they were being assessed for different reasons (one for sleep recording, one to map the scalp topography of his known epileptic focus). Twenty five (54\%) of the forty six patients had their diagnoses changed from the pre-monitoring diagnosis. Six (14\%) had their diagnoses confirmed. Fifteen (32\%) patients had inconclusive studies and it was recommended that repeat studies be done. The post-monitoring diagnoses can be seen in Table 3 . The most prevalent diagnosis was pseudoseizures, made in nineteen $(41 \%)$ patients. Fourteen of these were female. A number of patients had their seizures more accurately classified resulting in more efficacious therapy being applied. Two patients were diagnosed as having myoclonic seizures and have since had good results on valproate therapy. Another patient had been diagnosed as having partial complex seizures for years, and we were able to show that she in fact had typical absence seizures and absence status. She has had some improvement on ethosuximide therapy.

The patients diagnosed as pseudoseizures all had their anticonvulsant therapy discontinued if they were not also having 


\section{Table 3: Postmonitoring Diagnosis}

\begin{tabular}{lccc}
\hline \hline Seizure Type & Male & Female & Total \\
\hline Generalised tonic clonic & $1(2)^{*}$ & $0(0)$ & 0 \\
Partial complex & $5(10)$ & $3(6)$ & 8 \\
Myotonic & $0(0)$ & $2(4)$ & 2 \\
Absence & $0(0)$ & $1(2)$ & 1 \\
Pseudoseizures & $5(10)$ & $14(29)$ & 19 \\
Inconclusive & $7(15)$ & $8(17)$ & 15 \\
Not applicable & $2(4)$ & $0(0)$ & 2 \\
\hline Total & $20(42)$ & $28(58)$ & 48 \\
\hline
\end{tabular}

*Figures in brackets represent rounded percentages.

definite epileptic seizures. Some patients were observed in hospital for a period after medication was stopped. Some were subsequently referred for psychiatric evaluation. Selected patients were shown portions of the video recordings of their episodes.

Five patients had a prior diagnosis of epilepsy but had recently developed ictal events of a different character than those for which they had originally been diagnosed. We were able to demonstrate the presence of pseudoseizures in three of these patients. The other two had inconclusive studies.

The number of days of recording necessary to achieve a satisfactory diagnosis is variable. Approximately $40 \%$ of patients were diagnosed with less than four days recording (one $-4 \%$, two $-13 \%$, three $-20 \%$ ). The remainder required a full four day period, with one patient requiring two recording periods.

Ambulatory recordings were made using a four channel cassette recorder in one patient. This record was used in follow up to determine how successful therapy had been. The amount of spike and wave activity was quantitated over a twenty four hour period and a reduction was observed with therapy.

\section{Discussion}

The use of prolonged EEG and video monitoring equipment was of value in our centre in clarifying diagnoses and modifying treatment in a majority of the patients referred to us. These patients had undergone considerable prior investigation including multiple EEG recordings, and in hospital observation, without a satisfactory diagnosis being reached. We were able to make a firm diagnosis in $54 \%$ of the patients examined. In some this meant that complicated medication regimes could be eliminated.

It is interesting to compare our data to those of other centres. Quesney, Gloor and Andermann ${ }^{17}$ report that thirty of fifty patients monitored had partial complex seizures, the majority with lateralised EEG findings. These patients had all been selected as candidates for seizure surgery, and hence exhibit a degree of pre-selection bias. Stalberg ' made a satisfactory diagnosis on $65 \%$ of 185 recordings. Binnie et al ${ }^{2}$ achieved this with $57 \%$ of 99 recordings. These figures are comparable to ours.

In our series a large number of the patients were diagnosed as having pseudoseizures. This is probably due to a bias, as only patients not yet diagnosed were referred. Pseudoseizures were often suspected prior to monitoring, but the clinical information did not allow a firm diagnosis to be made.

The tapes made during monitoring provided an additional educational benefit not previously appreciated. The opportunity for house staff and nursing staff to observe both epileptic seizures and pseudoseizures in a relaxed retrospective fashion improved their understanding and management of these patients.
Brief ictal events often are not observed by traditional methods and more serious episodes are often viewed less objectively in real time. Subtle features are often missed but will be readily available to review on the monitoring tapes.

In order to set up an intensive monitoring program, a dedicated room is not necessary. Although a private room is preferable, the equipment is portable and can be set up wherever there is space and nursing supervision. It is essential that the nursing and house staff are instructed in the proper use of the equipment, and more specifically in how to alleviate problems such as tape malfunctions and camera adjustments. Constant supervision by an EEG technologist is not necessary.

The minimum length of recording time necessary for diagnostic accuracy is not clear. Many centres use short recordings of four to seven hours, or repetitive short recordings. . $^{1.7-9.11-13.15}$ Binnie et $\mathrm{al}^{2}$ recorded up to four days. The shorter recording time is certainly less expensive and recordings may be done on an outpatient basis. The longer recording period allows one to observe diurnal variations and gives the patient more time to become accustomed to the environment, thus more closely simulating natural conditions. It also allows controlled tapering of anticonvulsant medications and supervision of sleep deprivation.

There are a few caveats to consider with this technique. The patient must be cooperative; lack of co-operation may be a limiting factor in obtaining good quality recordings. The transmitter range is limited especially if there are obstructions in the recording area. The situation can be improved by experimenting with the placement of the receiving antenna. Interference from other electrical equipment did not appear to be a problem. When lighting conditions in the room change, the nursing staff must remember to adjust the camera or significant portions of the video tape will be unuseable.

The four channel ambulatory cassette recorder was used in follow up of one patient who had true absence seizures. A number of problems became apparent that would preclude using this device as the initial investigating instrument. Unless the patient is very reliable in keeping a $\log$, it is difficult to make good clinical and electrographic correlations. In addition, with only three channels of EEG it is difficult to detect artefact easily. ${ }^{23}$ At times the activity from eye movements would resemble spike and wave discharges. The video record helps to eliminate some of these problems with artefact. This device did prove useful in quantitating the effectiveness of therapy by allowing measurement of the amount of spike and wave activity in a 24 hour period.

In summary the technique of EEG and video monitoring has proven in our hands to be an effective diagnostic technique in the assessment of epilepsy. It has also proven to be a valuable staff and patient educational aid. The high number of inconclusive recordings may be reduced by repeated and longer recording times in selected patients. In addition the more uniform application of activation procedures, in particular discontinuing anticonvulsant medications may be of benefit. This technique was first utilised by centres evaluating patients for epilepsy surgery. Our data suggests that it need not be limited to this application.

\section{ACKNOWLEDGMENTS}

The authors would like to thank B. Lucas, M. DiPaolo, and N. Lane of the EEG department of Kingston General Hospital for their technical assistance. 


\section{REFERENCES}

1. Stalberg E. Experiences with long-term telemetry in routine diagnostic work. In: Kellaway P, Petersen I, eds. Quantitative analytic studies in epilepsy. New York: Raven Press, 1976: 269-278.

2. Binnie CD, Rowan AJ, Overweg J, et al. Telemetric EEG and video monitoring in epilepsy. Neurology 1981; 31: 298-303.

3. Ramani SV, Quesney LF, Olson D, et al. Diagnosis of hysterical seizures in epileptic patients. Am J Psychiatry 1980; 137: 705-709.

4. Holmes GL, McKeever M, and Russman BS. Abnormal behavior or epilepsy? Use of long-term EEG and video monitoring with severely to profoundly mentally retarded patients with seizures. Am J of Mental Deficiency 1983; 87: 456-458.

5. Holmes GL, Sackellares JC, McKiernan J, et al. Evaluation of childhood pseudoseizures using EEG telemetry and video tape monitoring. J of Pediatrics 1980; 97: 554-558.

6. Luther JS, McNamara JO, Carwile S, et al. Pseudoepileptic seizures: Methods and video analysis to aid diagnosis. Ann Neurol 1982; 12: 458-462.

7. Desai BT, Porter RJ, and Penry JK. Psychogenic seizures. A study of 42 attacks in six patients, with intensive monitoring. Arch Neurol 1982; 39: 202-209.

8. Ramani V, and Gumnit RJ. Management of hysterical seizures in epileptic patients. Arch Neurol 1982; 39: 78-81.

9. King DW, Gallagher BB, Murvin AJ, et al. Pseudoseizures: Diagnostic evaluation. Neurology 1982; 32: 18-23.

10. So EL, King DW, and Murvin AJ. Misdiagnosis of complex absence seizures. Arch Neurol 1984; 41: 640-641.

11. Iivanainen $M$. Intensive monitoring of intractable seizures. Acta Neurol Scand [Suppl] 1983; 93: 21-30.
12. Perry TR, Gumnit RJ, Gates JR, et al. Routine EEG vs. intensive monitoring in the evaluation of intractable epilepsy. Public Health Reports 1983; 98: 384-389.

13. Sutula TP, Sackellares JC, Miller JQ, et al. Efficacy of intensive monitoring and prolonged hospitalization in refractory epilepsy. Trans Am Neurol Assoc 1979; 104: 214-217.

14. Sutula TP, Sackellares JC, Miller JQ, et al. Intensive monitoring in refractory epilepsy. Neurology 1981; 31: 243-247.

15. Ramani V, and Gumnit RJ. Intensive monitoring of epileptic patients with a history of episodic aggression. Arch Neurol 1981; 38: 570-571.

16. Ramani V, and Gumnit RJ. Intensive monitoring of interictal psychosis in epilepsy. Ann Neurol 1981b; 11:613-622.

17. Quesney LF, Gloor P, and Andermann F. Indications for seizure monitoring. XX Canadian Congress of Neurological Sciences. Montreal Abstract. 1985; 13: 183.

18. McGrath DM. Video recording seizure activity in children. MCN 1983; 8: 218-220.

19. Mizrahi EM. Electroencephalographic/polygraphic/videomonitoring in childhood epilepsy. J of Pediatrics 1984; 105: 1-9.

20. Browne TR, Feldman RG. Epilepsy: An overview. In: Browne TR, Feldman RG, eds. Epilepsy. Diagnosis and management. Boston/Toronto: Little, Brown \& Co, 1983: 1-10.

21. Dreifuss FE. Proposal for revised clinical and electroencephalographic classification of epileptic seizures. Epilepsia 1981; 22: 489-496.

22. Williamson PD, Spencer DD, Spencer SS, et al. Complex partial seizures of frontal lobe origin. Ann Neurol 1985; 18: 497-504.

23. Ebersole JS, and Leroy RF. Evaluation of ambulatory cassette EEG monitoring: 1II. Diagnostic accuracy compared to intensive inpatient EEG monitoring. Neurology 1983; 33: 853-860. 